\title{
Biodiesel a partir de aceite usado de locales gastronómicos: efecto de la temperatura de reacción
}

Raul M. Saavedra ${ }^{1}$, Hernán J. García ${ }^{1}$, María I. Sánchez de Pinto y Mario D. Baigorí

\section{Resumen}

El objetivo del trabajo fue evaluar la producción de biodiesel mediante transesterificación alcalina de aceites usados (AU) recolectados en tres locales gastronómicos, a diferentes temperaturas de reacción. Los AU utilizados como materia prima provienen: bar-comedor de la UNSE-sede central(AUU), rotisería Santa Cruz(AUR) y bar-comedor de Villa Zanjón(AUZ). E1 índice de acidez de los aceites AUR y AUZ fue menor al $1 \%$ mientras que el del AUU fue de $1,4 \%$. La humedad se disminuyó a valores menores al $1 \%$. La obtención del biodiesel se realizó mediante transesterificación alcalina utilizando metanol e $\mathrm{KOH}$, a $120 \mathrm{~min}$ de reacción variando la temperatura entre 25,45 y $65^{\circ} \mathrm{C}$. Con los tres tipos de aceites y a las tres temperaturas de reacción el rendimiento fue superior al 95\% en casi todas las producciones. El punto de inflamación fue aumentando con la temperatura de reacción desde $98^{\circ} \mathrm{C}$ a $208^{\circ} \mathrm{C}$. Las características fisicoquímicas determinadas a los biodiesel cumplen con los parámetros aconsejados por las legislaciones vigentes. La utilización de AU en la cocción de alimentos para la producción de biodiesel permitiría producir biocombustible y resolver el problema ambiental que generan estos AU cuando son vertidos en cauces de agua o en pozos absorbentes sin un tratamiento previo adecuado.

Palabras claves: aceite usado, transesterificación, biodiesel, aguas de lavado.

\section{Abstract}

The objective of the work was to evaluate the production of biodiesel by

\footnotetext{
${ }^{1}$ Instituto de Ciencias Químicas. Facultad de Agronomía y Agroindustrias. UNSE. Av. Belgrano (S) 1912-CP4200. Santiago del Estero, Argentina.Tel: +54 385450958 - e-mail: raul_saav@hotmail.com

${ }^{2}$ Planta Piloto de Procesos Industriales Microbiológicos. Universidad Nacional de Tucumán. Avenida Belgrano y Pasaje Caseros - CP: T4001 MVB. Tucumán, Argentina.
} 
alkaline transesterification of used oils (UO) collected in three gastronomic stores, at different reaction temperatures. The UOs used as raw material come from: bar-dining room of the UNSE-central headquarters (AUU), Santa Cruz rotisserie $(A U R)$ and bar-dining room of Villa Zanjon (AUZ). The acidity index of the AUR and AUZ oils was less than $1 \%$ while that of the AUU was 1.4\%. The humidity was reduced to values lower than $1 \%$. The biodiesel was obtained by alkaline transesterification using methanol and $\mathrm{KOH}$, at 120 min of reaction, varying the temperature between 25,45 and $65^{\circ} \mathrm{C}$. With the three types of oils and the three reaction temperatures, the yield was over 95\% in almost all productions. The flash point was increased with the reaction temperature from $98^{\circ} \mathrm{C}$ to $208^{\circ} \mathrm{C}$. The physicochemical characteristics determined to biodiesel comply with the parameters recommended by the legislation in force. The use of the UO in the cooking of food for the production of biodiesel would allow to produce biofuel and solve the environmental problem generated by these UO when they are discharged in water channels or in absorbent wells without adequate prior treatment.

Keywords: oil used, transesterification, biodiesel, washing waters.

\section{Introducción}

El biodiesel ha sido identificado como una de las opciones que puede reemplazar al petróleo y sus derivados (o al menos complementarlo) disminuyendo el impacto ambiental que supone su procesamiento y consumo[1], [2]. Es biodegradable y se obtienen a partir de materias primas renovables las cuales se encuentran distribuidas a nivel global[3].

Para la producción de biodiesel, como materia prima, se están evaluando varias fuentes biológicas de origen renovables, tales como aceites vegetales y grasas[2], algunas de las cuales compiten con la alimentación humana, por lo que es necesaria la búsqueda de cultivos energéticos de oleaginosas no comestibles. En este sentido, los aceites vegetales usados (AVU) cobran vital importancia como materia prima disponible. E1 AVU es un residuo líquido que se generan luego de la fritura de alimentos, tanto en domicilios (D) como en locales gastronómicos (LG). En la ciudad de Santiago del Estero el 40\% de los LG consumen hasta $100 \mathrm{~L} / \mathrm{mes}$ de aceite vegetal (AV) y el $25 \%$ entre $100-200 \mathrm{~L} / \mathrm{mes}$ [4]. Considerando que existen centenares de LG que utilizan AV para las frituras de alimentos y que el AVU generado como residuo es el 50\% del aceite utilizado, se contaría con un volumen importante de AVU para ser valorizado como biocombustible [5], [4].

American Society for Testing and Materials (ASTM) define al "biodiesel" como un éster alquílico de ácidos grasos de cadena larga, obtenido por transesterificación de recursos renovables tales como de aceites vegetales o grasas animales [2], [6]. En la reacción de transesterificación, una molécula de triglicérido reacciona con tres moléculas de alcohol para dar tres moléculas de monoésteres (biodiesel) y una de gli- 
cerol [2]. El proceso de tranesterificacion depende de varios parámetros, tales como, materia prima, catalizador, alcohol, tiempo y temperatura de reacción, presión y velocidad de reacción [7]. El aumento de temperatura acelera la velocidad de reacción. Un límite de la temperatura es el punto de ebullición del alcohol utilizado. En el caso del metanol que tiene un punto de ebullición de $65-70^{\circ} \mathrm{C}$ la máxima temperatura de trabajo debería ser $60^{\circ} \mathrm{C}$. Una temperatura muy elevada no sólo favorecería la transesterificación sino también podría favorecer la saponificación, reduciendo el rendimiento [6]. Los biodiesel, para poder ser utilizados en el automotor diésel, deben cumplir los requisitos para los combustibles minerales de automoción dispuestos en las normas europeas EN-590, EN 14214, mientras que, para Argentina rige desde el 2004 la norma IRAM 6515-1 sobre los requisitos de calidad del FAME[8].

El objetivo del trabajo fue evaluar la producción de biodiesel mediante transes- terificación alcalina de aceites usados (AU) recolectados en tres locales gastronómicos, a diferentes temperaturas de reacción.

\section{Materiales y métodos}

Como materias primas se utilizaron AU recolectados en tres locales gastronómicos (LG): AUU (aceite usado bar-UNSE), AUR (aceite usado ROTISERÍA) y AUZ (aceite usado comedor-ZANJÓN). La metodología empleada fue la transesterificación alcalina de los AU con metanol en presencia de catalizador básico, a temperatura constante y agitación controlada. En el balón se colocaron $700 \mathrm{~mL}$ de $\mathrm{AU}$, $175 \mathrm{~mL}$ metanol (el 25\% del volumen de AU inicial) y como catalizador $\mathrm{KOH}$ cuya cantidad agregada dependió del contenido de acidez y densidad de los AU[6]. La producción de biodiesel se realizó a diferentes temperaturas de reacción: 25,45 y $65^{\circ} \mathrm{C}$ durante120 minutos de reacción. El equipo utilizado para la producción de biodiesel se muestra en la Figura 1.

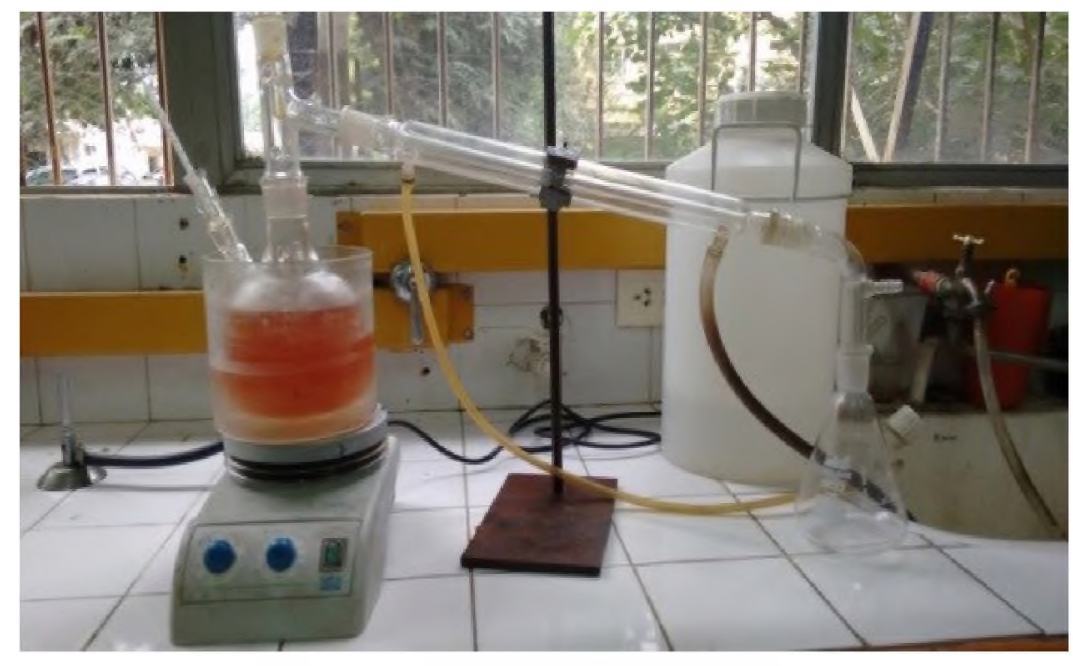

Figura 1.- Equipo de producción de biodiesel 
Para la caracterización fisicoquímica de aceites y biodiesel y para el control de calidad de los biodiesel obtenidos se emplearon las siguientes técnicas analíticas: contenido de humedad $(\mathrm{H})(\mathrm{mg} / \mathrm{Kg}$-ASTM $\mathrm{D} 4928)$, Índice de acidez (IA) ( $\mathrm{mgKOH} /$ $\mathrm{Kg}$-ASTM D 664), Densidad a $15^{\circ} \mathrm{C}$ (D) ( $\mathrm{g} / \mathrm{cm} 3$-ASTM D 4052), Viscosidad cinemática (VC) a $40^{\circ} \mathrm{C}(\mathrm{cSt}-\mathrm{ASTM}$ D445), Índice de refracción (IR) con refractómetro ATAGO ABBE DR-A1, Flash Point (FP) ( ${ }^{\circ} \mathrm{C}-\mathrm{ASTM}$ D93), Contenido de metanol (CMet) [8], Índice de corrosión al cobre (CCu) (ASTM-D-130).

\section{Resultados y discusiones}

3.1 Características de los aceites utiliza-

\section{dos como materia prima}

El color de los aceites recolectados varió de amarillo-marrón claro (AUZ) a amarillo-marrón oscuro (AUU). Existe una correlación entre el índice de acidez y las cantidades de ácidos grasos y di glicéridos presentes en los aceites usados, producto de la hidrólisis de los triglicéridos durante la cocción de los alimentos [9].

Tabla 1.- Características fisicoquímicas de los aceites utilizados como materia prima.

\begin{tabular}{|c|c|c|c|c|c|}
\hline Aceite & $\mathrm{D}(\mathrm{g} / \mathrm{mL})$ & $\mathrm{VC}(\mathrm{cSt})$ & $\mathrm{H}(\%)$ & $\mathrm{IA}(\%)$ & $\mathrm{IR}$ \\
\hline AUU & 0.91 & 66.91 & 0.57 & 1.40 & 1.4749 \\
\hline AUR & 0.92 & 66.92 & 0.53 & 0.99 & 1.4798 \\
\hline AUZ & 0.91 & 66.90 & 0.57 & 0.89 & 1.4795 \\
\hline
\end{tabular}

De los valores mostrados en la Tabla 1 se puede observar que: los valores de densidad, $0.91-0.92 \mathrm{~g} / \mathrm{mL}$ y los de viscosidad cinemática, $66.92 \mathrm{cSt}$, son similares en los tres aceites; los contenidos de humedad alcanzados luego del secado (0,53-0,57 \%) resultaron menores al $1 \%$. Valores superiores al $1 \%$ favorecen que durante la transesterificación alcalina, los ácidos grasos libres (AGL) reaccionen con el catalizador básico formando jabones[10], disminuyendo el rendimiento y afectando la purificación del biodiesel. Los aceites AUR y AUZ, poseen un indice de acidez inferior al $1 \%$, lo que indicaría que es favorable la producción de biodiesel por transesterificación alcalina en una sola etapa. Cuando el aceite posee un alto contenido de AGL, estos podrían reaccionar con el catalizador básico formando jabones, disminuyendo el rendimiento de la reacción e inhibiendo el posterior proceso de purificación del biodiesel, incluyendo la separación del glicerol y el agua de lavado. El indice de refracción (IR) está relacionado con el grado de saturación del aceite e indica la presencia de ácidos grasos insaturados de cadena larga, su valor varía entre 1.4749 y 1.4798 , similares a lo reportado en otros trabajos para este tipo de aceites.

3.2Producción de Biodiesel: efecto de la temperatura de reacción en el rendimiento de la reacción y características fisicoquímicas de los biodiesel obtenidos. 
De los resultados mostrados en la $\mathrm{Ta}-$ bla 2 , se observa que para los tres tipos de aceites y a las tres temperaturas de reacción evaluadas el rendimiento fue superior al $95 \%$ en todas las producciones, aumentando al $99 \%$ a las temperaturas superiores.

Se observa que (Tabla 2) durante la transesterificación se produzco una reducción de la viscosidad cinemática de los biodiesel respecto a la de los aceites utilizados como materia prima (Tabla 1), alcanzado en los biodiesel valores entre $3,65-4,78 \mathrm{cSt}$ manteniéndose los valores entre los límites aconsejados. Los valores del punto de inflamación o ignición de los biodiesel obtenidos resultaron superiores $120^{\circ} \mathrm{C}$, excepto para los BioAUZ obtenidos a $20-25^{\circ} \mathrm{C}$ y $40-45^{\mathrm{a}} \mathrm{C}$, observándose que a mayor temperatura de reacción mayor es el aumento del punto de inflamación, lo que implica además, una disminución del contenido de metanol sin reaccionar con el aumento de la temperatura de reacción. Los valores de corrosión al cobre y de indice de refracción no variaron con la temperatura de reacción y cumplen con los valores aconsejados.

Tabla 2.- Características físicoquímicas de los biodiesel BioAUJ, BioAUR y BioAUZ obtenidos a 120 min

\begin{tabular}{|c|c|c|c|c|c|c|c|c|c|c|}
\hline \multirow{3}{*}{$\begin{array}{l}\text { Parámetros } \\
\text { Fisicoquímicos }\end{array}$} & \multirow{2}{*}{\multicolumn{3}{|c|}{$\begin{array}{c}\text { BioAUU } \\
\text { Temp. de reacción }\left({ }^{\circ} \mathrm{C}\right)\end{array}$}} & \multirow{2}{*}{\multicolumn{3}{|c|}{$\begin{array}{c}\text { BioAUR } \\
\text { Temp. de reacción }\left({ }^{\circ} \mathrm{C}\right)\end{array}$}} & \multirow{2}{*}{\multicolumn{3}{|c|}{$\begin{array}{c}\text { BioAUZ } \\
\text { Temp. de reacción }\left({ }^{\circ} \mathrm{C}\right)\end{array}$}} & \multirow{3}{*}{$\begin{array}{c}\text { Limites } \\
\text { IRAM } \\
6515 \\
\text { EN } 14214\end{array}$} \\
\hline & & & & & & & & & & \\
\hline & $20 / 25$ & $40 / 45$ & $60 / 65$ & $20 / 25$ & $40 / 45$ & $60 / 65$ & $20 / 25$ & $40 / 45$ & $60 / 65$ & \\
\hline IA $(\%)$ & 0.56 & 0.57 & 0,87 & 0,28 & 0.33 & 0,32 & 0.34 & 0.28 & 0.38 & $<0,5$ \\
\hline $\mathrm{H}(\%)$ & 0.46 & 0.16 & 0,49 & 0,10 & 0.11 & 0,21 & 0.36 & 0.19 & 0.16 & $<0.05$ \\
\hline $\mathrm{D}(\mathrm{g} / \mathrm{mL})$ & 0.89 & 0.87 & 0,88 & 0,87 & 0.89 & 0,90 & 0.87 & 0.87 & 0.88 & $0.86-0.90$ \\
\hline $\mathrm{VC}(\mathrm{cSt})$ & 3.65 & 3.86 & 4.78 & 3,81 & 3,91 & 4,55 & 3.83 & 4.21 & 4.69 & $3.5-5.0$ \\
\hline $\mathrm{FP}\left({ }^{\circ} \mathrm{C}\right)$ & 136 & 168 & 208 & 166 & 170 & 198 & 98 & 118 & 204 & $>120$ \\
\hline CMet & 0.12 & 0.08 & 0.06 & 0.09 & 0.08 & 0.06 & 0.21 & 0.15 & 0.06 & $<0.2$ \\
\hline $\mathrm{CCu}$ & $1 \mathrm{a}$ & $1 \mathrm{a}$ & $1 \mathrm{a}$ & $1 \mathrm{a}$ & $1 \mathrm{a}$ & $1 \mathrm{a}$ & $1 \mathrm{a}$ & la & $1 \mathrm{a}$ & $<n^{\circ} 3$ \\
\hline IR & 1.45 & 1.45 & 1,46 & 1.45 & 1.45 & 1,46 & 1.45 & 1.45 & 1.45 & $1.45<1.46$ \\
\hline Rto $(\%)$ & 97,9 & 98,2 & 99,0 & 95,7 & 96,7 & 99,7 & 98,6 & 99,3 & 99.1 & \\
\hline
\end{tabular}

\section{Conclusiones}

Los biodiesel obtenidos presentan las características de calidad aconsejadas por la normativa vigente para su utilización como biocombustible en motores diésel.

E1 rendimiento de reacción fue superior al $95 \%$ en todas las producciones, aumentando al $99 \%$ a las temperaturas superiores. Los puntos de inflamación aumentaron con el aumento de la temperatura de reacción, alcanzado valores superiores a $200^{\circ} \mathrm{C}$.

\section{Referencias}

[1]J. Chen, R. D. Tyagi, J. Li, X. Zhang, P. Drogui, and F. Sun, "Economic assessment of biodiesel production from wastewater sludge," Bioresour. Technol., vol. 253, no. January, pp. 41-48, 2018.

[2]S. A. García-Muentes, C. F. Lafargue-Pérez, C. B. Labrada-Vázquez, C. M. Díaz-Velázquez, and C. A. E. Sánchez del Campo-Lafita, "Propiedades fisicoquímicas del aceite y biodiesel producidos de la Jatropha curcas L en la provincia de Manabí , Ecuador," Rev. 
Cuba. Química, vol. 30, no. 1, pp. 142-158, 2018.

[3]P. A. Ramos Rodríguez, A. Milán Hernández, E. A. Espinosa Melo, L. M. Zumalacárregui de Cárdenas, L. Pérez Ruíz, and R. Piloto-Rodríguez, "Caracterización del biodiesel obtenido del aceite de Jatropha curcas L," Afinidad, vol. 75, no. 581, p. 6(45-51), 2018.

[4]R. M. Saavedra, H. J. García, P. M. Juárez, M. I. Sánchez de Pinto, and M. D. Baigorí, "Aceite usado en locales gastronómicos y domicilios: comportamiento ciudadano," in Ambiente y desarrollo sostenible desde una perspectiva multidisciplinaria, III Congreso de Ciencia y Tecnología Ambiental - Argentina y Ambiente 2017. ISBN 978-98746096-3-2, 2017, p. 6 (206-211).

[5]Y. Zhang, X. Bao, G. Ren, X. Cai, and J. Li, "Analysing the status, obstacles and recommendations for WCOs of restaurants as biodiesel feedstocks in China from supply chain' perspectives," Resour. Conserv. Recycl., vol. 60, pp. 20-37, 2012.

[6]R. M. Saavedra, P. M. Juárez, H. J. García, M. I. Sánchez de Pinto, and A. N. Ardila,
"Biodiesel a partir de aceite usado domiciliario: efecto del tiempo de reacción," Investigaciones en Facultades de Ingeniería del NOA (ISSN: 1853-6662), vol. 3, Catamarca. Argentina, p. 7 (321-328), 2017.

[7]P. J. García-Moreno, M. Khanum, A. Guadix, and E. M. Guadix, "Optimization of biodiesel production from waste fish oil," Renew. Energy, vol. 68, pp. 618-624, 2014.

[8]R. M. Saavedra, H. J. García, P. M. Juárez, E. Jorge de Cuba, M. İ. Sanchez de Pinto, and M. D. Baigorí, "Aceite usado en locales gastronoómicos: comportamiento ciudadano y producción de biodiesel," in III Congreso Argentino de Ingenieria, III Congreso Argentino de Ingeniería. ISBN 9789504201731, 2016, p. 13(2326-2338).

[9]F. J. Fajardo Hernández, “Técnicas de Caracterización para el Biodiésel basadas en la Normatividad Internacional ASTM," 2015.

[10]M. K. Lam, K. T. Lee, and A. R. Mohamed, "Homogeneous, heterogeneous and enzymatic catalysis for transesterification of high free fatty acid oil (waste cooking oil) to biodiesel: A review," Biotechnol. Adv., vol. 28 , no. 4, pp. 500-518, 2010. 